\title{
Raynaud's of the tongue following chemoradiation for squamous cell carcinoma of the oropharynx
}

\author{
AMIT MITTAL $^{1}$ and BHARAT B. MITTAL $^{2}$ \\ ${ }^{1}$ Department of Dermatology, Yale University School of Medicine, New Haven, CT 06510; ${ }^{2}$ Department of Radiation \\ Oncology, Northwestern University, Feinberg School of Medicine, Chicago, IL 60611, USA
}

Received August 12, 2016; Accepted November 9, 2016

DOI: $10.3892 / \mathrm{mco} .2016 .1103$

\begin{abstract}
Raynaud's of the tongue following radiation alone or chemoradiotherapy (CRT) is a rare occurrence. The present study reports a case where typical symptoms and signs of Raynaud's phenomenon involving the tongue occurred $\sim 18$ months following CRT treatment in a 53-year-old female, who was a smoker prior to CRT with stage $\mathrm{T}_{2} \mathrm{~N}_{2} \mathrm{cM} 0$ local-regional advanced stage IV oropharyngeal cancer. The patient was treated using cisplatin chemotherapy and intensity-modulated radiation with a dose of 70 Gray (Gy). The intermittent episodes of painful discoloration of the tongue were exacerbated due to the cold and emotional stress. No definite clinical or laboratory evidence of connective tissue disorder was identified. To the best of our knowledge, this is the first reported case of Raynaud's of the tongue following CRT without primary Raynaud's of the digits. The possible pathogenesis involving vascular and neural mechanism is discussed in the case report.
\end{abstract}

\section{Case report}

A 54-year-old female with a 20 pack-year smoking history, idiopathic peripheral neuropathy, hypercholesterolemia and polymyalgia was treated with concomitant cisplatin administered at $100 \mathrm{mg} / \mathrm{m}^{2}$ every 3 weeks for 3 cycles and prescribed doses of 70 Gray (Gy) radiation at 2 Gy per fraction/5 days a week for 7 weeks chemoradiotherapy (CRT) for a $\mathrm{T}_{2} \mathrm{~N}_{2} \mathrm{cM} 0$ [according to the tumor-lymph nodes-metastasis (TNM) staging system], p16-negative squamous cell cancer of the base of the tongue. At $\sim 18$ months following CRT, the patient experienced tingling of the tongue, numbness with white discoloration of the tongue, and difficulty in speaking (Fig.1A). Following these symptoms, the tongue turned bright

Correspondence to: Dr Bharat B. Mittal, Department of Radiation Oncology, Northwestern University, Feinberg School of Medicine, 420 East Superior Street, Chicago, IL 60611, USA

E-mail: bmittal@nm.org

Key words: Raynaud's, tongue, chemotherapy, radiation, oropharynx red, concomitantly with the onset of severe pain. Shortly afterwards, the pain subsided and the tongue returned to its normal color. The entire episode lasted 90-120 sec (Fig. 1B). Laboratory testing revealed that the patient's complete blood count (CBC), numbers of platelets, electrolytes, rheumatoid factor, 24-h urine cortisol, cyclic citrullinated peptide immunoglobulin G ( $I g G)$, thyroid function test and erythrocyte sedimentation rate (ESR) were within the normal limits. The patient was positive for antinuclear antibody (ANA), although ANA-8 proved to be negative. C-reactive protein was increased to a level of $8.9 \mathrm{mg} / \mathrm{dl}$. During the two most recent years, intermittent episodes of painful discoloration have persisted, particularly during cold weather and in times of emotional stress; otherwise, the patient is well. The rheumatology department of Northwestern University was consulted and, due to characteristic symptoms and discoloration, a diagnosis of Raynaud's of the tongue was made. The patient has no history of Raynaud's affecting her digits, and she refused to take steroids, vasodilators or calcium-channel blockers.

\section{Discussion}

Raynaud's disease is a disorder of blood flow. Due to vasoconstriction, when exposed to cold or emotionally stressful situations, the affected area turns white or blue, followed by tingling and throbbing pain, with color returning to normal after the blood flow has returned. The pathophysiology of primary Raynaud's has yet to be fully elucidated (1), although secondary Raynaud's is known to be caused by underlying connective tissue disease, injury or medication. Herrick et al (2) have described the pathogenesis according to three categories of abnormality: Vascular, neural and intravascular.

Raynaud's phenomenon of the tongue is a rare condition, although it has been shown to occur in patients with a prior history of primary Raynaud's of the extremities, with or without connective tissue disorders (3-5). An association among radiation, chemotherapy and Raynaud's has been reported.In patients with pre-existing primary Raynaud's of the digits, developing Raynaud's of the tongue and lips following radiation alone for oropharyngeal and lip cancer was first reported by Westbury et al (6). This group hypothesized that radiation-induced Raynaud's is most likely due to late injury caused to the capillaries and vasculature, with the primary target being endothelium. Additionally, radiation-induced 

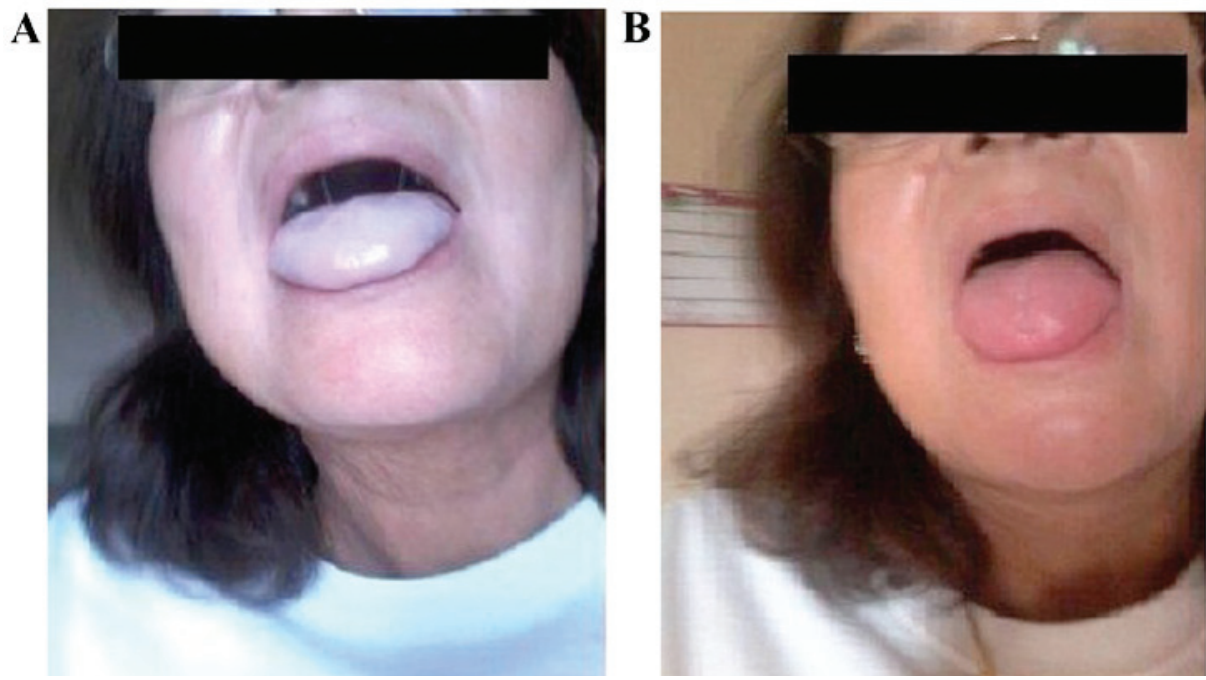

Figure 1. Appearance of the tongue during and after Raynaud's episode. (A) White discoloration of the tongue during an acute phase of Raynaud's phenomenon. (B) Normal coloration following the end of Raynaud's episode.

damage to vascular media and adventitia results in fibrosis. The end result is the structural and functional alteration of blood vessels, impairment of chemical mediators such as nitric oxide, hypoxia, and altered vascular response to cold stimuli.

Cisplatin alone or in combination with other chemotherapeutic agents has been shown to induce Raynaud's phenomenon. Mohokum et al (7) performed a meta-analysis of 24 studies published between 1981 and 2010, and concluded that, despite the heterogeneity among the studies, patients who received cisplatin-based chemotherapy had a greater incidence of Raynaud's. The exact mechanism of this association has yet to be fully elucidated. It is possibly associated with cisplatin-induced hypomagnesemia, given the important roles mediated by magnesium in maintenance of vascular smooth muscle tone (8), vascular ischemia (9) and the autonomic nervous system (10).

In our patient, the symptoms of Raynaud's were likely to have been due to vascular and neural toxicity from CRT. The symptoms appeared $\sim 18$ months following the completion of CRT. At two years following the first appearance of symptoms, the patient is doing well, despite intermittent episodes of Raynaud's of the tongue. To the best of our knowledge, Raynaud's of the tongue following CRT without primary Raynaud's of the digits has not previously been reported. That the occurrence of this phenomenon is so rare may result in delayed diagnosis and intervention.

\section{References}

1. Herrick AL: The pathogenesis, diagnosis and treatment of Raynaud phenomenon. Nat Rev Rheumatol 8: 469-479, 2012.

2. Herrick AL, Illingworth K, Blann A, Hay CR, Hollis S and Jayson MI: Von Willebrand factor, thrombomodulin, thromboxane, beta-thromboglobulin and markers of fibrinolysis in primary Raynaud's phenomenon and systemic sclerosis. Ann Rheum Dis 55: 122-127, 1996.

3. Giunta JL: Raynaud disease with oral manifestations. Arch Dermatol 111: 78-80, 1975.

4. Katada Y and Tanaka T: Images in clinical medicine. Lingual Raynaud's phenomenon. N Engl J Med 366: e12, 2012.

5. Cohen JC, Palomba ML and Morris LG: Raynaud's phenomenon of the tongue. J Rheumatol 40: 336, 2013.

6. Westbury CB, Harrington KJ, Rhys-Evans P, Archer DJ, Searle AE, Henk JM, Black CM and Nutting CM: Raynaud's phenomenon after radical radiotherapy for tumours of the head and neck. Postgrad Med J 79: 176-177, 2003.

7. Mohokum M, Hartmann P and Schlattmann P: The association of Raynaud's syndrome with cisplatin-based chemotherapy - a meta-analysis. Eur J Intern Med 23: 594-598, 2012.

8. Vogelzang NJ, Bosl GJ, Johnson K and Kennedy BJ: Raynaud's phenomenon: A common toxicity after combination chemotherapy for testicular cancer. Ann Intern Med 95: 288-292, 1981.

9. Stefenelli T, Kuzmits R, Ulrich W and Glogar D: Acute vascular toxicity after combination chemotherapy with cisplatin, vinblastine, and bleomycin for testicular cancer. Eur Heart J 9: 552-556, 1988.

10. Pliarchopoulou K and Pectasides D: Late complications of chemotherapy in testicular cancer. Cancer Treat Rev 36: 262-267, 2010. 